\title{
Serial optical coherence tomography for characterization of coronary lithotripsy efficacy: How much is enough?
}

\author{
Maksymilian P. Opolski, Jacek Kwiecinski, Rafal Wolny, \\ Artur Debski, Adam Witkowski \\ Department of Interventional Cardiology and Angiology, \\ National Institute of Cardiology, Warsaw, Poland
}

Intravascular lithotripsy (IVL) is an emerging treatment for severely calcified lesions. A 74-year-old female with a history of diabetes and chronic kidney disease presented with the Canadian Cardiovascular Society class III angina. Coronary angiography demonstrated a heavily calcified tandem stenosis in the mid right coronary artery (RCA) (Fig. 1A, Suppl. Video 1). Following predilatation with 1.25 to $2.5 \mathrm{~mm}$ semi-compliant balloons and $3.0 \mathrm{~mm}$ noncompliant balloon, the baseline optical coherence tomography (OCT) using saline flush media (as prevention for contrast-induced nephropathy) revealed extensive three-quadrant calcium in the mid RCA (Fig. 1B-C). A $3.5 \times 12 \mathrm{~mm}$ lithotripsy balloon was subsequently inflated to $4 \mathrm{~atm}$ and 20 pulses of sonic pressure (in both lesions in mid RCA) were applied (40 pulses in total), followed by first post-IVL OCT showing deep calcium fracture with significant luminal gain (Fig. 1D, E). After successful delivery of consecutive 20 pulses in each of the two stenoses (80 pulses in total), the second post-IVL OCT demonstrated no further modification of calcification (Fig. 1F). Ultimately, a $3.5 \times 38 \mathrm{~mm}$ drug-eluting stent was implanted followed by post-dilatation with $4.0 \mathrm{~mm}$ non-compliant balloon resulting in good angiographic and OCT result (Fig. 1G-I).

The association between calcium disruption and the specified dosage of mechanical pulses generated by IVL catheter is unknown. The present case demonstrates extensive calcium fractures along with a significant luminal gain following the first 20 pulses of IVL within a balloon-resistant lesion. Interestingly, further applications of IVL (up to 40 pulses per lesion) did not translate into additional calcium modification. This observation provides insights into the efficiency of IVL.

Conflict of interest: None declared

Address for correspondence: Dr. Maksymilian P. Opolski, Department of Interventional Cardiology and Angiology, National Institute of Cardiology, ul. Alpejska 42, 04-628 Warsaw, Poland. tel: +48501444303, fax: +48(22)6133819, e-mail: opolski.mp@gmail.com

Received: 15.04.2020 Accepted: 5.05.2020 


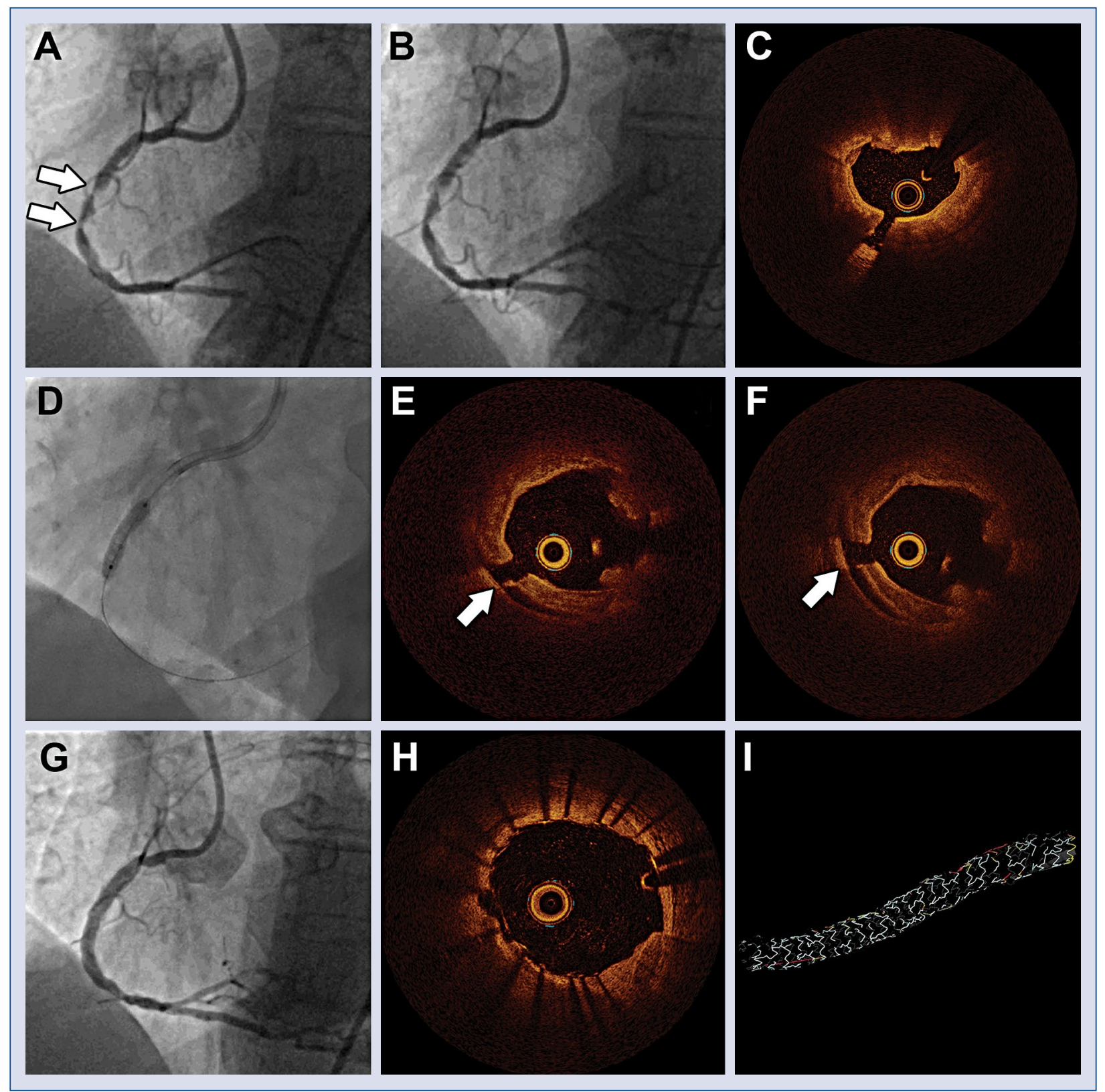

Figure 1. A, B. Invasive angiography showing heavily calcified tandem stenosis (arrows) of the mid-right coronary artery before (A) and after (B) non-compliant balloon predilatation; C. Optical coherence tomography after non-compliant balloon predilatation showing three-quadrant calcification; D. Angiographic appearance of the intravascular lithotripsy balloon; E, F. Optical coherence tomography after successful delivery of 20 pulses (E) and 40 pulses (F) of intravascular lithotripsy revealing extensive calcium fracture (arrow); G-I. Final angiography (G) and optical coherence tomography $(\mathbf{H}-\mathbf{I})$ showing optimal stent expansion and apposition. 